\title{
Reviewers for The Journal of the American Board of Family Practice in 1997
}

The following individuals participated in the peer review of manuscripts submitted to this journal during 1997. Their contributions are gratefully acknowledged.

Acheson, Louise

Alexander, Elizabeth

Allen, Deborah

Baird, Mac

Berg, Alfred O.

Bergman, James J.

Blackman, James

Brody, Howard

Brown, Richard

Brucker, Paul

Brummel-Smith, Kenneth

Campbell, Thomas

Cass, Alvah

Chester, Beth

Chestnut, Charles

Clover, Richard

Coombs, John

Crouch, Michael

Culpepper, Larry

David, Allen

Davidson, Robert C.

Demers, Raymond

Eiff, Patrice

Ellsbury, Kathleen

Ellsworth, Allan

English, Eugenia

Fletcher, Robert

Fowkes, William

Frame, Paul

Frey, Keith

Galazka, Sim

Garry, Joseph

Green, Larry

Hagen, Michael

Heidrich, Fred

Herter, Christian

Kahn, Arlo
Katon, Wayne

Kirchner, Jeffrey

Larsen, Lars

Leversee, Jack

Lillington, Glenn A.

Magill, Michael

McBride, Patrick

Mengel, Mark

Millard, Peter S.

Neighbor, William

Nesbitt, Thomas S.

Norris, Thomas E.

Nuovo, James

Phillips, Ted

Phillips, William

Prislin, Michael

Rabinowitz, Howard

Ramsey, Scott

Realini, Janet

Rodriguez, Glenn S.

Ruane, Thomas

Scherger, Joseph

Schneeweiss, Ronald

Schwenk, Thomas

Sloane, Philip

Spach, David

Spann, Stephen

Stephens, G. Gayle

Stevens, Nancy

Sugarman, Jonathan

Sullivan, Sean

Taplin, Stephen

Taylor, Thomas

Thom, David

Toewe, Clinton $\mathrm{H}$.

Wall, Eric

Young, Paul 